\title{
Etiology and Risk Factors of Acute and Chronic Pancreatitis
}

\author{
Frank Ulrich Weiss Felix Laemmerhirt Markus M. Lerch \\ Department of Medicine A, Greifswald Medical School, Greifswald, Germany
}

Keywords

Pancreatitis - Etiologic factors · Alcohol abuse - Genetic risk

\section{Abstract}

Based on the recognition of common etiological and genetic risk factors, acute and chronic pancreatitis are increasingly regarded as a continuum of the same disease, with a significant overlap of clinical manifestations and phenotypes but distinct morphological and imaging appearances. Recent population-based and cohort studies have found that tobacco smoke conveys a greater risk than immoderate alcohol consumption for the development of chronic pancreatitis, and hypertriglyceridemia has been identified as a risk factor for acute pancreatitis - even when plasma levels are only mildly elevated. Hereditary pancreatitis, in its autosomal dominant form, is associated with mutations in the cationic trypsinogen gene (PRSS1), whereas a number of germline variations in other genes have been found to represent risk factors for chronic as well as acute pancreatitis. For now, most of these involve the pancreatic digestive protease/antiprotease system. Oftentimes, affected patients are burdened with multiple or accumulating risk factors, and genetic traits when combined with environmental toxins compound the chance of developing the disease. Determining the underlying etiology of pancreatitis is worth the effort since formerly intractable varieties such as autoimmune pancreatitis are now becoming increasingly treatable, and subtype-specific therapeutic modalities may become available.

(c) 2019 S. Karger AG, Basel

\section{Introduction}

Pancreatitis, an inflammatory condition of the pancreas, is generally not caused by infectious agents, but triggered by initiating factors like gallstones and immoderate alcohol consumption. Complex gene-environment interactions may trigger or affect the pathogenesis and course of the disease (see Table 1). The widely accepted paradigm that acute, recurrent, and chronic pancreatitis are different disease entities has been replaced by the concept of a disease continuum: $30 \%$ of patients with acute pancreatitis will develop a chronic disease form, often with an overlap as recurrent pancreatitis in the years between. This review focuses on the etiologic factors that associate with the different disease phenotypes and the triggering factors involved in the progression from acute to chronic disease.

\section{Acute Pancreatitis: Definition and Etiology}

With an incidence of 13-45/100,000 acute pancreatitis is one of the most common ICD diagnoses for contacting emergency services and for hospitalization in Europe and the USA [1]. Based on the revised Atlanta classification acute pancreatitis is defined by two of three criteria - typical belt-like abdominal pain, elevated serum lipase level three times above the normal threshold, or radiological imaging signs of pancreatitis. Every year 54,000 patients with acute pancreatitis are treated in German hospitals [2]. Based on the total number of pancreatitis patients no gender predominance is found, albeit male sex is more often associated with an alcoholic etiology, whereas wom-

Dr. Frank Ulrich Weis

Klinik und Poliklinik für Innere Medizin A, Universitätsmedizin Greifswald Ferdinand-Sauerbruch-Strasse

DE-17489 Greifswald (Germany)

E-Mail ulrich.weiss@ uni-greifswald.de 
Table 1. Overview of known risk factors of acute pancreatitis, their involved pathomechanism, and the associated risk for chronicity of the disease

\begin{tabular}{lll}
\hline Risk factors for acute pancreatitis & Assumed pathomechanism & Risk for disease chronicity \\
\hline Alcohol abuse & dose-dependent cell injury & high* \\
Smoking & dose-dependent cell toxicity & high* \\
Gallstones & pancreatic duct obstruction & - \\
Hypertriglycerides & blood viscosity, local tissue ischemia & high (in familial hypertriglyceridemia) \\
Endoscopic procedures (ERCP) & cell injury, increased duct pressure & - \\
Abdominal trauma & pancreatic tissue damage & high (for disrupted duct syndrome) \\
Drugs & cell toxic or metabolic effects & - \\
Autoimmune diseases & immune cell infiltration & high \\
Dominant PRSS1 mutations & elevated trypsin activity & very high \\
Predisposing genetic mutations & protease activation, ER stress & high \\
Infections & direct tissue injury & - \\
\hline
\end{tabular}

Of note, complex gene-environment interactions may constitute the individual risk. ER, endoplasmic reticulum. ${ }^{*}$ In cases of longpersisting abuse.

en tend to have more often biliary pancreatitis. The peak incidence of alcoholic acute pancreatitis in woman is between 25 and 34 years and in men 10 years later [3]. The overall pancreatitis risk, which includes all etiologies, increases continuously with age. Typically, individuals are affected in their sixth decade of life [4]. Black people have a two- to three-fold elevated pancreatitis risk compared to whites [5]. The mortality depends on the subtype of acute pancreatitis. Mild, edematous pancreatitis shows a mortality of only $1 \%$, whereas the severe, necrotizing form is associated with a death toll of up to $25 \%$ [3]. Characteristically, $20-30 \%$ of patients with acute pancreatitis experience recurrent pancreatitis attacks and of these $10 \%$ develop chronic pancreatitis.

\section{Etiology of Acute Pancreatitis \\ Gallstones}

Gallstones and alcohol abuse are the most common causes of acute pancreatitis and each account for the underlying etiology in $30-50 \%$ of cases. Cross-sectional studies of pancreatitis patients demonstrate that $50 \%$ of women and only $15 \%$ of men have stones in the gallbladder, explaining why the female sex predominates the biliary etiology of pancreatitis. Up to $20 \%$ of an adult population carries gallstones [6,7]. Either stones in the gallbladder or in the biliary tract predispose to pancreatitis. While $75 \%$ of gallbladder stone carriers remain asymptomatic, $8 \%$ of patients with gallstones will ultimately develop acute pancreatitis [8]. Often, acute pancreatitis is the first manifestation of biliary stones, independent of their original location. While the majority of patients with biliary acute pancreatitis recover completely after a mild edematous pancreatitis episode, $15-30 \%$ develop severe necrotizing pancreatitis, requiring intensive care and multidisciplinary treatment strategies. Gallstones trigger acute pancreatitis when they become impacted at the duodenal papilla and obstruct the outflow from the pancreatic duct $[9,10]$. This leads to increased pancreatic pressure, sometimes only transiently, but induces acinar cell injury and triggers the disease onset [10].

\section{Alcohol in Acute Pancreatitis}

Next to gallstones, alcohol is the most common factor associated with acute pancreatitis. For more than a century alcohol has been known as an etiology of pancreatitis and it is now well established that immoderate alcohol consumption can initiate an episode of acute pancreatitis and increase the susceptibility to chronic pancreatitis. The peak incidence age of alcohol-associated acute pancreatitis is between 35 and 44 years in men and between 25 and 34 years in women [2]. It was postulated that consumption of between 50 and $80 \mathrm{~g}$ or $4-7$ drinks per day injure the gland, although individual differences must be considered. While the association between alcohol and pancreatitis is epidemiologically evident, only a minority of alcoholics ever develop acute or chronic pancreatitis. This implies that alcohol consumption alone is rarely the sole precipitating factor for pancreatitis, rather alcohol sensitizes the pancreas for co-factors such as a high lipid diet, infectious agents, or tobacco smoke, the latter being an independent and probably stronger risk factor than alcohol alone - at least for chronic pancreatitis. Alcohol is eliminated from the body by various metabolic processes. The primary enzymes involved are aldehyde dehydrogenase (ALDH), alcohol dehydrogenase (ADH), cytochrome P450 (CYP2E1), and catalase. Variations in the genes for these enzymes have been found to influence alcohol consumption, alcohol-related tissue damage, and alcohol dependence. Based on a developmental relation, the pancreas, like the liver, can metabolize alcohol, and 
both oxidative and non-oxidative pathways are functional in the pancreas. Via the oxidative pathway ADH and CYP2E1 metabolize ethanol to acetaldehyde and reactive oxygen species. Metabolites of the nonoxidative ethanol metabolism are fatty ester ethyl esters which can harm the gland [11]. Triggered by ethanol and its metabolites, pancreatic acini undergo complex changes in cellular homeostasis like intracellular calcium levels [12], endoplasmic reticulum stress, increase of mitochondrial permeability [13], impairment in autophagy, or co-localization and activation of lysosomal and pancreatic digestive enzymes [14].

\section{Hypertriglyceridemia}

The incidence of acute pancreatitis is related to elevated triglycerides in an estimated 10\% of cases [15]. Most subjects with elevated triglycerides experience no symptoms. It is assumed that elevated plasma levels of triglycerides and chylomicrons increase the blood viscosity leading to local ischemia in tissue [13]. During tissue ischemia, the metabolism changes from aerobic to anaerobic and cells depend upon anaerobic glycolysis, the final product of which is L-lactate. Local ischemia elicits lactate levels and acidosis; the latter increases the toxicity of free fatty acids and is the premise for autoactivation of trypsinogen. In combination with other risk factors like alcohol, tobacco, or pharmaceutical drugs ischemia can turn into a local inducer of acute pancreatitis [16]. Patients with familial lipoprotein lipase deficiency have an idiosyncratic risk for recurrent episodes of acute pancreatitis [17]. Also, pregnant women have an inherent risk for hypertriglyceridemia-induced acute pancreatitis, based on a hormone triggered elevation of cholesterol and triglyceride levels [18]. Specific differences have been reported between primary and secondary hypertriglyceridemia. The Fredrickson classification distinguishes between five types of primary hyperlipidemia, of which types I, IV, and $\mathrm{V}$ are associated with an increased risk for acute pancreatitis. Type I is mainly characterized by elevated chylomicrons like in lipoprotein lipase deficiency and type IV shows high very-low-density lipoproteins (VLDLs), whereas type $\mathrm{V}$ has increased plasma levels of both VLDL and chylomicrons. Primary hypertriglyceridemia is based on genetic aberrations with recessive (type I) or dominant (type IV and V) autosomal inheritance [19, 20]. Secondary hypertriglyceridemia is associated with obesity, pregnancy, insufficiently controlled diabetes, medications, or chronic and acute alcohol abuse. According to the Atlanta classification system the diagnosis of hypertriglyceridemia-induced acute pancreatitis requires a serum triglyceride level of over $1,000 \mathrm{mg} / \mathrm{dL}$. The elevated risk for the development of at least 1 attack of acute pancreatitis is $\sim 20 \%$ in a population with triglyceride levels above $1,000 \mathrm{mg} / \mathrm{dL}$ [19]. Murphy et al. [21] reported a risk in- crease of $4 \%$ for acute pancreatitis with every rise of 100 $\mathrm{mg} / \mathrm{dL}$ serum triglycerides, even at elevations much lower than $1,000 \mathrm{mg} / \mathrm{dL}$.

\section{Endoscopic Procedures}

Pathologies of the pancreatic or biliary tract are frequent indications for performing endoscopic retrograde cholangiopancreatography (ERCP). A feared complication of ERCP is the induction of post-ERCP acute pancreatitis. Severe post-ERCP pancreatitis is a rare event ( $\sim .5 \%$ of pancreatitis cases, $5 \%$ of ERCPs), but compared with other etiologies the severity is often high. The reasons for the post-ERCP acute pancreatitis include patient-related, operator-related, and procedure-related factors. Sex, age, anomalies of the sphincter Oddi or the gland, like pancreas divisum, preexisting pancreatitis, and biliary pancreatitis as an indication for ERCP, are patient-related factors. Operator-related complications are associated with the experience of the endoscopist and overlap with procedure-related complications. A difficult cannulation of the papilla Vateri may lead to edema, sphincter spasm, and pancreatic duct obstruction [22]. The osmolarity, $\mathrm{pH}$, and composition of the contrast medium my contribute to chemical and hydrostatic damage. Equally, the increased pressure while injecting the contrast media can cause the activation of digestive enzymes which then trigger pancreatic autodigestion and the initiation of local inflammation [23].

\section{Trauma}

The incidence of abdominal trauma in poly-traumatized patients is $20 \%$ in Germany; $95 \%$ of these cases involve blunt force trauma. Usually parenchymatous organs like the liver and spleen are affected, and traumatic injury to the pancreas is rare (6\%) due to its retroperitoneal location. Still, there is no association between the force effect and the extent of pancreatic damage. Common consequences of traumatic damage to the pancreas are pancreatitis, fissures, lacerations, and a disconnected duct, or a combination of them, which already indicates the complexity of management for traumatic pancreatic injuries. Abdominal traumata are associated with a high mortality, the reason being a potential for severe bleeding, septic complications, and an increased rate of multi-organ failure [24].

\section{Rare Causes}

The diagnosis of medication-induced acute pancreatitis is rare (0.1-2\%). In the context of polypharmacy of an aging population the number of drug-induced cases of pancreatitis will rise and may be underestimated. The pathomechanism is largely drug-specific but can include sphincter spasm, cytotoxic and metabolic effects, hypersensitivity reactions, localized angioedema, or arteriolar 
thrombosis $[25,26]$. The diagnosis is laborious and based on ruling out other, more common etiologies. Commonly prescribed drugs which are known to be associated with acute pancreatitis are angiotensin-converting enzyme (localized angioedema), statins (direct and accumulation toxicity), oral contraceptives or hormone replacement therapy, especially estrogen (hypertriglyceridemia, local arteriolar thrombosis), diuretics, antiviral therapy (HIV), valproic acid, and antidiabetic agents such as GLP-1 mimetic [25-28]. Interestingly, while it was shown that being the carrier of $\mathrm{ABO}$ blood type $\mathrm{B}$ increases the risk of developing chronic pancreatitis [29], a recent study has found that the same association exists for drug-induced (i.e., azathioprine-induced) acute pancreatitis in patients with inflammatory bowel disease [30].

\section{Recurrent Acute and Chronic Pancreatitis: Distinction and Etiology}

Recurrent acute pancreatitis is characterized by relapsing episodes of acute pancreatitis and morphological signs of a normal gland in asymptotic intervals [31]. The diagnosis of recurrent acute pancreatitis is made retrospectively after the second episode of acute pancreatitis. Often, signs of chronic pancreatitis are found after the first attack of pancreatitis or during follow-up, suggesting that the junction between recurrent and chronic pancreatitis is fluent [32]. Sankaran et al. [33] point out in a systematic review that the risk of recurrence after a first attack of acute pancreatitis is $20 \%$, and $35 \%$ of patients with at least 1 recurrence will progress to chronic pancreatitis. While acute pancreatitis befalls patients in their sixth decade of life, individuals with recurrent acute pancreatitis are younger and diagnosed between 30 and 40 years of age with a predominance of the male sex. Andersson et al. [34] demonstrated in a retrospective study of a single Swedish hospital that patients with admission for acute pancreatitis were 5 years older than patients with a recurrent subtype. The difference in distribution of age and sex in patients with recurrent pancreatitis compared with those with a single event of acute pancreatitis is related to the underlying etiologies (less gallstones, more intensive drinking behavior). Younger patients in particular show a higher risk of alcohol-induced recurrent pancreatitis and chronicity based on continued alcohol consumption.

\section{Chronic Pancreatitis}

Chronic pancreatitis has an incidence of 4-14/100,000 per year and a prevalence of $13-52 / 100,000[2,35]$. Chronic pancreatitis is defined as a continuous inflammatory degeneration of the exocrine and endocrine pancreatic tissue with irreversible morphological changes [36]. Typical morphological alterations include areas of inflammation, calcium deposits, duct changes, or pseudocysts. Next to an exocrine (steatorrhea, weight loss) and endocrine (type IIIc diabetes) insufficiency, recurrent pain attacks agonize patients with chronic pancreatitis [37]. Patients with chronic pancreatitis are predominantly male und diagnosed between the fifth and sixth decade of life. The peak incidence of acute alcohol-induced pancreatitis is a decade earlier than for chronic alcohol-related pancreatitis $[2,3]$. In hereditary pancreatitis, a rare form which is associated with autosomal dominant mutations in the cationic trypsinogen gene (PRSS1), the disease starts in childhood and progresses through variable clinical recurrences to chronic pancreatitis in the second or third decade of life [38]. While acute idiopathic pancreatitis shows an increasing incidence with age which plateaus after 65 years independently of the sex, chronic idiopathic pancreatitis is characterized by a dual age distribution. Early-onset idiopathic chronic pancreatitis appears during the third decade of life, while a lateonset subtype peaks during the sixth to seventh decade $[2$, $3,39]$.

\section{Alcohol in Chronic Pancreatitis}

Alcohol abuse is the most common etiology associated with chronic pancreatitis, which accounts for approximately $65 \%$ of all cases. In 2009 , a systematic review and meta-analysis analyzed the association between alcohol consumption and pancreatitis and found a monotonically increasing dose-response relationship [40]. The doseresponse relationship between alcohol and chronic disease typically varies by sex, with women experiencing higher risks at comparatively lower levels of intake [41]. There are individual variations between the duration of abuse and the amount of daily consumed alcohol until the first attack of pancreatitis or the grade of pancreatic impairment. The pathomechanism of alcohol-induced injury of the pancreatic gland is not fully understood. One theory proposes that oxidative stress induced by alcohol metabolites damage the gland directly. Long-term abuse induces the alcohol detoxifying enzyme cytochrome $\mathrm{P} 450$ $2 \mathrm{E} 1$ in pancreatic acinar cells, increasing acetate within the pancreas and inhibiting cellular processes and defense mechanisms [42, 43]. Although it is a minor pathway, fatty ester ethyl esters formed by nonoxidative elimination of ethanol harm the gland and are produced to a greater extent in the pancreas than in other organs [11]. Another theory rests in the experimental and clinical observation that ethanol has a direct effect on fluid secretion from the pancreatic duct via impairment of the cystic fibrosis transmembrane regulator [44]. Although there is strong association between alcohol abuse and pancreatitis, relatively few individuals who abuse alcohol develop chronic pancreatitis. This fact indicates that alcoholic pancreatitis is not caused by alcohol abuse alone $[45,46]$. Instead, the 
Fig. 1. Pancreatitis-associated genetic risk factors (sequence variants) influence activation, auto-activation, inhibition, or degradation processes of proteases and change the protease/anti-protease equilibrium in pancreatic acinar cells.

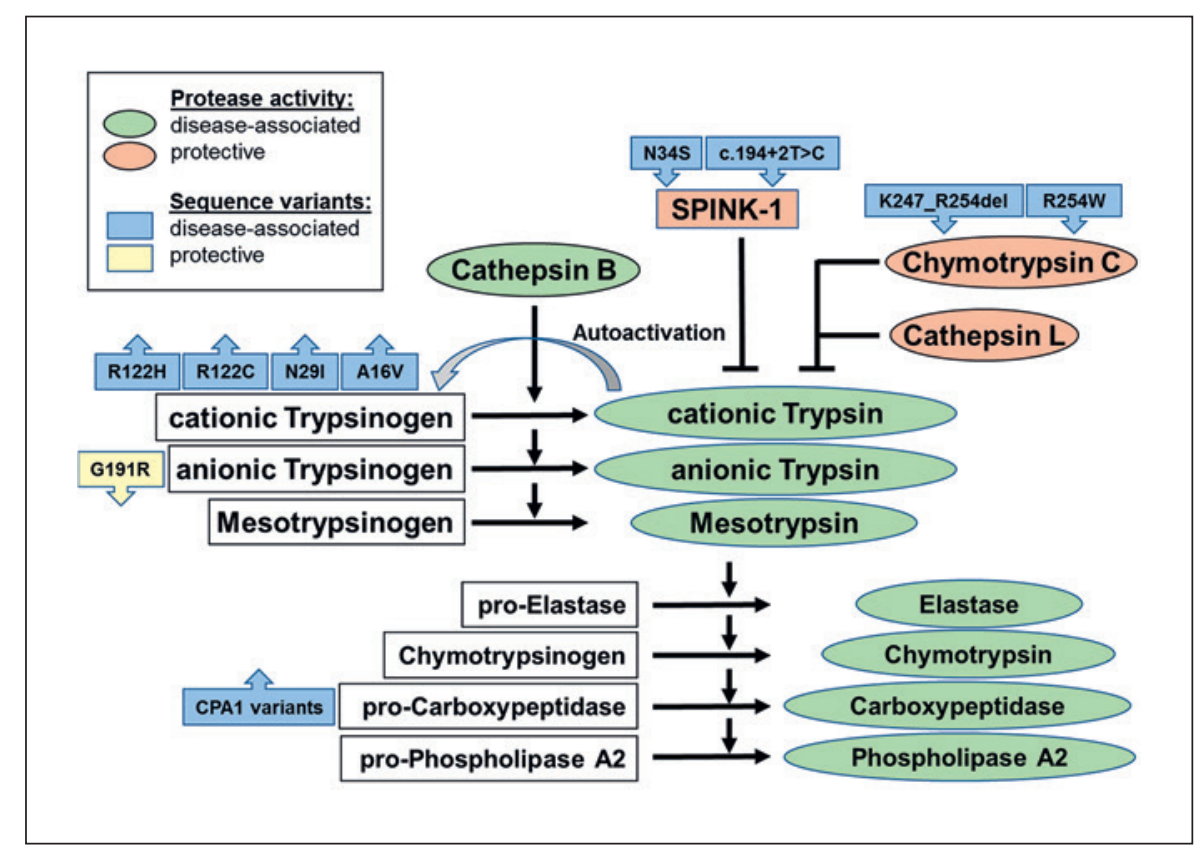

pancreas may be sensitized to injury by alcohol consumption, and external or environmental factors stimulate the initiation of the disease. A number of mechanisms by which ethanol and both its oxidative and nonoxidative metabolites damage pancreatic cells are currently discussed. Several factors are believed to be involved in the pathogenesis including genetic predisposition, high lipid diet, cigarette smoking, and infectious agents [47].

\section{Genetic Risk}

Genetic variants in several genes have been identified as being associated with susceptibility to chronic pancreatitis and replicated in multiple cohorts [38, 48, 49]. The most relevant genes include the cationic trypsinogen gene (PRSS1), the pancreatic secretory trypsin inhibitor gene (SPINK1), the cystic fibrosis transmembrane conductance regulator gene (CFTR), the chymotrypsinogen gene $(C T R C)$, and the carboxy peptidase A1 gene (CPA1). These major genetic susceptibility factors were identified as candidate genes linked to intrapancreatic trypsin activity regulation within the pancreas or reduced ductal fluid flow $[44,50]$. Within the "trypsin-dependent pathological pathway" variants in susceptibility genes PRSS1, SPINK1, and CTRC increase pancreatitis risk by promoting harmful trypsinogen activation or by impairing protective trypsinogen degradation and/or trypsin inhibition (Fig. 1). Activation of PRSS1 trypsinogen to active trypsin within the pancreas is responsible for disease onset and progression. Protective mechanisms to control trypsinogen activation include trypsin inhibition by SPINK1 and trypsinogen degradation by chymotrypsin $\mathrm{C}(C T R C)$ and trypsin. Other PRSS1 variants, such as the activation peptide mutants p.D22G, p.K23R, and p.K23_I24insIDK, are clinically rare and tend to associate primarily with sporadic idiopathic disease. These mutants all exhibit reduced secretion because trypsinogen becomes intracellularly autoactivated and degraded [51-53]. It appears likely that inside the endoplasmic reticulum active trypsin is sensed as a misfolded state of trypsinogen, which prompts degradation. In addition, the intracellular levels of the misfolded protein should depend on how efficiently the cell is capable of disposing them. Mutations in digestive enzymes that induce misfolding and endoplasmic reticulum stress represent a highly relevant pathological concept and a potential therapeutic target in chronic pancreatitis. Mutations in PRSS1 and CPA1 are the best studied examples. Strong risk factors are occasionally associated with hereditary forms of pancreatitis.

Significant association with pancreatitis has also been demonstrated for sequence variants in CASR [54], CLDN2 [55-57], and CEL [58, 59]. Preliminary reports which await further validation include CTSB [60], MYO9B [61], and UBR1 [62], or the association of an increased pancreatitis risk with $\mathrm{ABO}$ blood group and the so-called "secretor status," which is determined by a mutation of the fucosyltransferase gene FUT2 [29, 63]. Additional genetic variants are being evaluated and will likely be added to this list in the future. A single factor alone rarely causes pancreatitis, and the majority of patients with recurrent acute and chronic pancreatitis carry multiple variants in one or multiple genes, coupled with environmental stressors. So far only PRSS1 mutations are inherited in an autosomal dominant pattern, giving rise to a hereditary form of pancreatitis that runs in families. Other genetic variants, as well as different environmental factors, can be strong risk factors for susceptibility and 

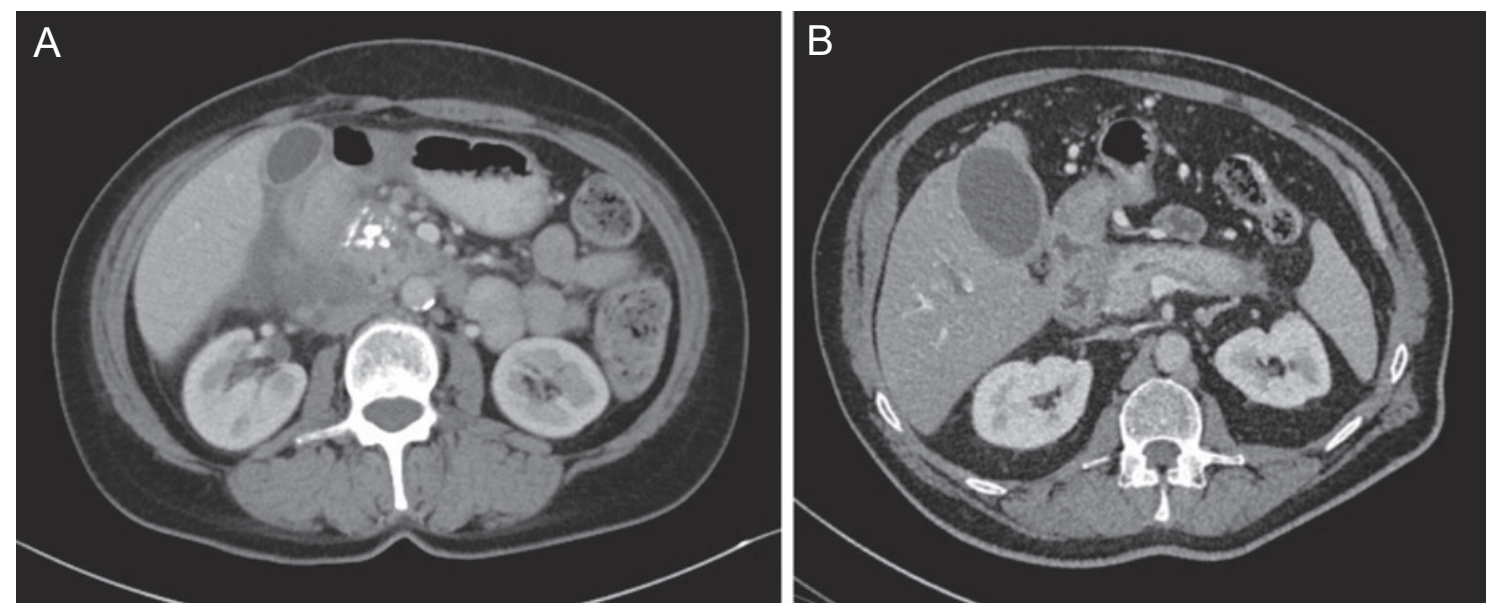

Fig. 2. A CT scan of patient with SPINK1-p.N34S/CTRC-p.G60G mutation. Note enlargement of the head of the pancreas and multiple calcifications. B CT scan of patient with autoimmune pancreatitis type 1 (IgG4 related) with a homogenous (sausage-shaped) enlargement of the entire gland and an enhanced rim.

progression of acute pancreatitis, recurrent acute pancreatitis, and chronic pancreatitis. In a multinational crosssectional study (INSPPIRE consortium) of 301 children with recurrent acute and chronic pancreatitis $84 \%$ of children with chronic pancreatitis reported prior recurrent episodes of acute pancreatitis [64]. Sequencing analysis identified at least one mutation in pancreatitis-related genes in $48 \%$ of patients with recurrent acute pancreatitis versus $73 \%$ of patients with chronic pancreatitis. Children with PRSS1 or SPINK1 mutations were more likely to develop chronic pancreatitis, but ethnic differences also seemed to influence the disease phenotype as well as disease progression.

Genome-wide association analyses and next generation sequencing studies are powerful new technologies that are capable of identifying genetic risk factors outside of candidate gene-driven screening approaches. A few genome-wide association studies have been performed to date, mainly in chronic pancreatitis and recurrent acute pancreatitis patients. Studies by Whitcomb et al. [55] and Rosendahl et al. [65] were able to identify new susceptibility loci in the claudin-2 gene (CLDN2), as well as a gene conversion event in the chymotrypsin B CTRB1-2 locus, which modifies the risk for alcoholic and nonalcoholic chronic pancreatitis [65].

\section{Hereditary Pancreatitis}

Hereditary pancreatitis is a rare autosomal dominant genetic disorder of the pancreas with incomplete penetrance. In most cases recurrent episodes of acute pancreatitis start in childhood (median age of onset, 10 years) [66]. Chronic pancreatitis develops in young adulthood and results in an elevated risk of pancreatic cancer beginning in the fifth decade of life [48]. Typically, hereditary pancreatitis is associated with a limited number of gain- of-function mutations in the cationic trypsinogen gene (PRSS1), the most common of which are p.A16V, p.N29I, and p.R122H. The clinical features are similar to those seen in other causes of chronic pancreatitis. Primary manifestations are abdominal pain, maldigestion as a consequence of pancreatic exocrine dysfunction, and diabetes mellitus due to islet cell damage. In a national series of 418 patients with hereditary pancreatitis from 112 families in 14 European countries, exocrine pancreatic insufficiency eventually developed in $37 \%$ and diabetes mellitus eventually developed in $48 \%$ of cases [38]. The most common presentation of autosomal dominant hereditary pancreatitis is recurrent acute pancreatitis. However, some patients may present with chronic pancreatitis without experiencing prior episodes of acute pancreatitis.

\section{Rare Causes}

The pancreas divisum or annulare are congenital abnormalities which have been associated with chronic pancreatitis. Usually these congenital aberrations do not cause chronic pancreatitis by themselves. A second risk factor is required like smoking, alcohol abuse, or genetic predisposition. A biliary pathogenesis for chronic pancreatitis is an extremely rare event and based either on noncompliance concerning a needed cholecystectomy or an underdeveloped medical infrastructure. In rare cases pancreatitis may be caused by viral infections such as mumps, coxsackie B, mycoplasma pneumonia, and campylobacter, but these are mostly acute and not chronic episodes. In approximately 5-6\% of patients, chronic pancreatitis is caused by autoimmune inflammation [67]. Symptoms can be mild, but half of the patients with autoimmune pancreatitis (type 1) show elevated levels of immunoglobulin G4 (IgG4) and rarely develop pancreatic calcifications $[68,69]$ (Fig. 2). Additional character- 
istics of autoimmune pancreatitis include main pancreatic duct changes, scarring of the pancreatic tissue, and presence of elevated numbers of inflammatory cells. Autoimmune pancreatitis can occur by itself or in association with other autoimmune diseases such as primary sclerosing cholangitis, primary biliary cholangitis, retroperitoneal fibrosis, rheumatoid arthritis, sarcoidosis, and Sjögren's syndrome [70].

\section{Cancer Risk}

Chronic pancreatitis and carcinoma of the pancreas are equally common in Western countries. Incidence rates for both are around 10 per 100,000 [71]. The incidence rates for pancreatic cancer in both sexes increase with age, the highest being in the age group over 70 years [72]. Pancreatic cancer is predominantly a disease of the elderly, and almost $90 \%$ of all cases are diagnosed after the age of 55 years [72]. Chronic pancreatitis is regarded as a precancerous lesion in this context. It is generally accepted that persisting inflammation can cause repeated DNA damage and a progressive accumulation of genetic mutations. A sequential accumulation of genetic defects has been suggested to associate in the pancreas with precancerous histologic changes. These pancreatic intra-epithelial neoplasms (PanIN) are present in sporadic pancreatic adenocarcinomas as well as in patients with a history of chronic pancreatitis. Histologically, PanINs progress through stages of increasing architectural and cytological atypia, starting from low-grade dysplasia (PanIN-1A, PanIN-1B) to moderate dysplasia (PanIN-2) to highgrade dysplasia (PanIN-3). The first genetic mutations seen in the early stages include K-RAS mutations, followed by p16/CDKN2A, TP53, and SMAD4/DPC4 [73]. Mutations in all four genes have been recognized as driver mutations that trigger neoplastic transformation and tumor progression [74]. Importantly, chronic pancreatitis rarely develops into cancer, even not in the very longstanding cases, and the majority of patients presenting with pancreatic carcinoma have no history of chronic pancreatitis (less than $1 \%$ in population-based studies). The prognosis of chronic pancreatitis depends on the age at diagnosis and continuing alcohol or tobacco abuse.

\section{Conclusion}

Pancreatitis is a syndrome of various causes, symptoms, and outcomes [75]. Our understanding of the natural history of the disease and how treatment can effectively limit acute attacks or prevent disease progression and chronicity is still limited. Long-term follow-up and well-designed, preferably randomized, studies with adequate sample size are needed to improve our understanding of how complex gene-environment interactions shape the disease phenotype. Also, next generation sequencing technologies can elucidate the genetic risk outside of the established candidate gene-driven screening approaches. Both will improve our understanding of the pathophysiology of pancreatitis and help to develop new and successful therapeutic concepts for the benefit our patients.

\section{Acknowledgments}

The work of F.U.W. and M.M.L. is supported by grants from Deutsche Krebshilfe / Dr. Mildred-Scheel-Stiftung (109102), the Deutsche Forschungsgemeinschaft (DFG GRK 1947/A3), the Federal Ministry of Education and Research (BMBF GANI-MED 03IS2061A, 0314107, 01ZZ9603, 01ZZ0103, 01ZZ0403, 03ZIK012, and FKZ: 01EK1511A), and the European Union (EU-FP-7: EPCTM, V-630-S-150-2012/132/133, ESF/14-BM-A55-0045/16, TBIV-242-VBW-084, and TBI-V-1-245-VBW-085).

\section{Disclosure Statement}

In reference to this review, all authors have no conflicts of interest to report.

\section{References}

1 Peery AF, Crockett SD, Barritt AS, Dellon ES, Eluri S, Gangarosa LM, et al. Burden of Gastrointestinal, Liver, and Pancreatic Diseases in the United States. Gastroenterology. 2015 Dec;149(7):1731-1741.e3.

2 Yadav D, Timmons L, Benson JT, Dierkhising RA, Chari ST. Incidence, prevalence, and survival of chronic pancreatitis: a populationbased study. Am J Gastroenterol. 2011 Dec; 106(12):2192-9.

3 Lankisch PG, Assmus C, Maisonneuve P, Lowenfels AB. Epidemiology of pancreatic diseases in Lüneburg County. A study in a defined german population. Pancreatology. 2002;2(5):469-77.
4 Yadav D, Lowenfels AB. Trends in the epidemiology of the first attack of acute pancreatitis: a systematic review. Pancreas. 2006 Nov; 33(4):323-30.

5 Yang AL, Vadhavkar S, Singh G, Omary MB. Epidemiology of alcohol-related liver and pancreatic disease in the United States. Arch Intern Med. 2008 Mar;168(6):649-56.

6 Völzke H, Baumeister SE, Alte D, Hoffmann W, Schwahn C, Simon P, et al. Independent risk factors for gallstone formation in a region with high cholelithiasis prevalence. Digestion. 2005;71(2):97-105.
7 Buch S, Schafmayer C, Völzke H, Seeger M, Miquel JF, Sookoian SC, et al. Loci from a genome-wide analysis of bilirubin levels are associated with gallstone risk and composition. Gastroenterology. 2010 Dec;139(6): 19421951.e2.

8 European Association for the Study of the Liver (EASL). Electronic address: easloffice@ easloffice.eu. EASL Clinical Practice Guidelines on the prevention, diagnosis and treatment of gallstones. J Hepatol. 2016 Jul;65(1): 146-81.

9 Hernández CA, Lerch MM. Sphincter stenosis and gallstone migration through the biliary tract. Lancet. 1993 May;341(8857):1371-3. 
10 Rünzi M, Saluja A, Lerch MM, Dawra R, Nishino H, Steer ML. Early ductal decompression prevents the progression of biliary pancreatitis: an experimental study in the opossum. Gastroenterology. 1993 Jul;105(1): 157-64.

11 Huang W, Booth DM, Cane MC, Chvanov M, Javed MA, Elliott VL, et al. Fatty acid ethyl ester synthase inhibition ameliorates ethanolinduced $\mathrm{Ca} 2+$-dependent mitochondrial dysfunction and acute pancreatitis. Gut. 2014 Aug;63(8):1313-24

12 Saluja AK, Dawra RK, Lerch MM, Steer ML CCK-JMV-180, an analog of cholecystokinin, releases intracellular calcium from an inositol trisphosphate-independent pool in rat pancreatic acini. J Biol Chem. 1992 Jun;267(16): 11202-7.

13 Weidenbach H, Lerch MM, Gress TM, Pfaff D, Turi S, Adler G. Vasoactive mediators and the progression from oedematous to necrotising experimental acute pancreatitis. Gut. 1995 Sep;37(3):434-40.

14 Lerch MM, Saluja AK, Dawra R, Saluja M, Steer ML. The effect of chloroquine administration on two experimental models of acute pancreatitis. Gastroenterology. 1993 Jun; 104(6):1768-79.

15 Papachristou GI, Machicado JD, Stevens T, Goenka MK, Ferreira M, Gutierrez SC, et al. Acute pancreatitis patient registry to examine novel therapies in clinical experience (APPRENTICE): an international, multicenter consortium for the study of acute pancreatitis Ann Gastroenterol. 2017;30(1):106-13

16 Scherer J, Singh VP, Pitchumoni CS, Yadav D. Issues in hypertriglyceridemic pancreatitis: an update. J Clin Gastroenterol. 2014 Mar; 48(3):195-203.

17 de Pretis N, Amodio A, Frulloni L. Hypertriglyceridemic pancreatitis: Epidemiology, pathophysiology and clinical management. United European Gastroenterol J. 2018 Jun; 6(5):649-55.

18 Jin J, Yu YH, Zhong M, Zhang GW. Analyzing and identifying risk factors for acute pancreatitis with different etiologies in pregnancy. J Matern Fetal Neonatal Med. 2015 Feb 28(3):267-71.

19 Lloret Linares C, Pelletier AL, Czernichow S, Vergnaud AC, Bonnefont-Rousselot D, Levy $\mathrm{P}$, et al. Acute pancreatitis in a cohort of 129 patients referred for severe hypertriglyceridemia. Pancreas. 2008 Jul;37(1):13-2.

20 Brahm A, Hegele RA. Hypertriglyceridemia Nutrients. 2013 Mar;5(3):981-1001.

21 Murphy MJ, Sheng X, MacDonald TM, Wei L. Hypertriglyceridemia and acute pancreatitis. JAMA Intern Med. 2013 Jan;173(2):1624.

22 Fluhr G, Mayerle J, Weber E, Aghdassi A, Simon $\mathrm{P}$, Gress T, et al. Pre-study protocol MagPEP: a multicentre randomized controlled trial of magnesium sulphate in the prevention of post-ERCP pancreatitis. BMC Gastroenterol. 2013 Jan;13(1):11

23 Noble MD, Romac J, Vigna SR, Liddle RA. A $\mathrm{pH}$-sensitive, neurogenic pathway mediates disease severity in a model of post-ERCP pancreatitis. Gut. 2008 Nov;57(11):1566-71.
24 Nast-Kolb D, Trupka A, Ruchholtz S, Schweiberer L. [Abdominal trauma]. Unfallchirurg. 1998 Feb;101(2):82-91. German.

25 Nitsche C, Maertin S, Scheiber J, Ritter CA, Lerch MM, Mayerle J. Drug-induced pancreatitis. Curr Gastroenterol Rep. 2012 Apr; 14(2):131-8.

26 Kaurich T. Drug-induced acute pancreatitis. Proc Bayl Univ Med Cent. 2008 Jan;21(1):7781.

27 Alves C, Batel-Marques F, Macedo AF. A meta-analysis of serious adverse events reported with exenatide and liraglutide: acute pancreatitis and cancer. Diabetes Res Clin Pract. 2012 Nov;98(2):271-84

28 Thisted H, Jacobsen J, Munk EM, Nørgaard B, Friis S, McLaughlin JK, et al. Statins and the risk of acute pancreatitis: a population-based case-control study. Aliment Pharmacol Ther. 2006 Jan;23(1):185-90.

29 Weiss FU, Schurmann C, Guenther A, Ernst F, Teumer A, Mayerle J, et al. Fucosyltransferase 2 (FUT2) non-secretor status and blood group $\mathrm{B}$ are associated with elevated serum lipase activity in asymptomatic subjects, and an increased risk for chronic pancreatitis: a genetic association study. Gut. 2015 Apr; 64(4):646-56.

30 Teich N, Bokemeyer B, Mohl W, Walldorf J, Bruns T, Schmidt C, et al.; German IBD Study Group. Blood group B is associated with azathioprine-induced acute pancreatitis in patients with IBD. Gut. 2017 Aug;66(8):1531-2.

31 Sarles H, Sarles JC, Camatte R, Muratore R, Gaini M, Guien C, et al. Observations on 205 confirmed cases of acute pancreatitis, recurring pancreatitis, and chronic pancreatitis. Gut. 1965 Dec;6(6):545-59.

32 Testoni PA. Acute recurrent pancreatitis: Etiopathogenesis, diagnosis and treatment. World J Gastroenterol. 2014 Dec;20(45): 16891-901.

33 Sankaran SJ, Xiao AY, Wu LM, Windsor JA, Forsmark CE, Petrov MS. Frequency of progression from acute to chronic pancreatitis and risk factors: a meta-analysis. Gastroenterology. 2015 Nov;149(6):1490-1500.e1.

34 Andersson B, Andrén-Sandberg A, Nilsson J, Andersson R. Survey of the management of acute pancreatitis in surgical departments in Sweden. Scand J Gastroenterol. 2012 Sep; 47(8-9):1064-70.

35 Hoffmeister A, Mayerle J, Beglinger C, Büchler MW, Bufler P, Dathe K, et al.; Chronic Pancreatitis German Society of Digestive and Metabolic Diseases (DGVS). [S3-Consensus guidelines on definition, etiology, diagnosis and medical, endoscopic and surgical management of chronic pancreatitis German Society of Digestive and Metabolic Diseases (DGVS)]. Z Gastroenterol. 2012 Nov;50(11): 1176-224. German.

36 Lerch MM, Stier A, Wahnschaffe U, Mayerle J. Pancreatic pseudocysts: observation, endoscopic drainage, or resection? Dtsch Arztebl Int. 2009 Sep;106(38):614-21.

37 Mayerle J, Hoffmeister A, Werner J, Witt H, Lerch MM, Mössner J. Chronic pancreatitisdefinition, etiology, investigation and treatment. Dtsch Arztebl Int. 2013 May;110(22): 387-93.
38 Howes N, Lerch MM, Greenhalf W, Stocken DD, Ellis I, Simon P, et al.; European Registry of Hereditary Pancreatitis and Pancreatic Cancer (EUROPAC). Clinical and genetic characteristics of hereditary pancreatitis in Europe. Clin Gastroenterol Hepatol. 2004 Mar;2(3):252-61

39 Layer P, Yamamoto H, Kalthoff L, Clain JE, Bakken LJ, DiMagno EP. The different courses of early- and late-onset idiopathic and alcoholic chronic pancreatitis. Gastroenterology. 1994 Nov;107(5):1481-7.

40 Irving HM, Samokhvalov AV, Rehm J. Alcohol as a risk factor for pancreatitis. A systematic review and meta-analysis. JOP. $2009 \mathrm{Jul}$; 10(4):387-92.

41 Shield KD, Parry C, Rehm J. Chronic diseases and conditions related to alcohol use. Alcohol Res. 2013;35(2):155-73.

42 Bhopale KK, Falzon M, Ansari GA, Kaphalia BS. Alcohol oxidizing enzymes and ethanolinduced cytotoxicity in rat pancreatic acinar AR42J cells. In Vitro Cell Dev Biol Anim. 2014 Apr;50(4):373-80

43 Cartmell MT, Schulz HU, O’Reilly DA, Yang BM, Kielstein V, Dunlop SP, et al. Cytochrome P450 2E1 high activity polymorphism in alcohol abuse and end-organ disease. World J Gastroenterol. 2005 Nov;11(41) 6445-9.

44 Maléth J, Balázs A, Pallagi P, Balla Z, Kui B, Katona M, et al. Alcohol disrupts levels and function of the cystic fibrosis transmembrane conductance regulator to promote development of pancreatitis. Gastroenterology. 2015 Feb;148(2):427-39.e16.

45 Apte MV, Pirola RC, Wilson JS. Mechanisms of alcoholic pancreatitis. J Gastroenterol Hepatol. $2010 \mathrm{Dec} ; 25(12): 1816-26$.

46 Aghdassi AA, Weiss FU, Mayerle J, Lerch MM, Simon P. Genetic susceptibility factors for alcohol-induced chronic pancreatitis. Pancreatology. 2015 Jul;15(4 Suppl):S23-31.

47 Sata N, Koizumi M, Nagai H. Alcoholic pancreatopathy: a proposed new diagnostic category representing the preclinical stage of alcoholic pancreatic injury. J Gastroenterol. 2007 Jan;42(S17 Suppl 17):131-4.

48 Whitcomb DC, Gorry MC, Preston RA, Furey $\mathrm{W}$, Sossenheimer MJ, Ulrich CD, et al. Hereditary pancreatitis is caused by a mutation in the cationic trypsinogen gene. Nat Genet. 1996 Oct;14(2):141-5.

49 Keim V, Bauer N, Teich N, Simon P, Lerch MM, Mössner J. Clinical characterization of patients with hereditary pancreatitis and mutations in the cationic trypsinogen gene. Am J Med. 2001 Dec;111(8):622-6.

50 Weiss FU, Simon P, Bogdanova N, Mayerle J, Dworniczak B, Horst J, et al. Complete cystic fibrosis transmembrane conductance regulator gene sequencing in patients with idiopathic chronic pancreatitis and controls. Gut. 2005 Oct;54(10):1456-60.

51 Kereszturi E, Sahin-Tóth M. Intracellular autoactivation of human cationic trypsinogen mutants causes reduced trypsinogen secretion and acinar cell death. J Biol Chem. 2009 Nov;284(48):33392-9. 
52 Joergensen MT, Geisz A, Brusgaard K, Schaffalitzky de Muckadell OB, Hegyi P, Gerdes $\mathrm{AM}$, et al. Intragenic duplication: a novel mutational mechanism in hereditary pancreatitis. Pancreas. 2011 May;40(4):540-6.

53 Geisz A, Hegyi P, Sahin-Tóth M. Robust autoactivation, chymotrypsin $\mathrm{C}$ independence and diminished secretion define a subset of hereditary pancreatitis-associated cationic trypsinogen mutants. FEBS J. 2013 Jun; 280(12):2888-99.

54 Masson E, Chen JM, Férec C. Overrepresentation of Rare CASR Coding Variants in a Sample of Young French Patients With Idiopathic Chronic Pancreatitis. Pancreas. 2015 Aug;44(6):996-8.

55 Whitcomb DC, LaRusch J, Krasinskas AM, Klei L, Smith JP, Brand RE, et al.; Alzheimer's Disease Genetics Consortium. Common genetic variants in the CLDN2 and PRSS1PRSS2 loci alter risk for alcohol-related and sporadic pancreatitis. Nat Genet. 2012 Dec; 44(12):1349-54.

56 Derikx MH, Kovacs P, Scholz M, Masson E, Chen JM, Ruffert C, et al.; PanEuropean Working group on Alcoholic Chronic Pancreatitis Members and Collaborators. Polymorphisms at PRSS1-PRSS2 and CLDN2MORC4 loci associate with alcoholic and non-alcoholic chronic pancreatitis in a European replication study. Gut. 2015 Sep;64(9): 1426-33.

57 Weiss FU, Hesselbarth N, Párniczky A, Mosztbacher D, Lämmerhirt F, Ruffert C, et al. Common variants in the CLDN2-MORC4 and PRSS1-PRSS2 loci confer susceptibility to acute pancreatitis. Pancreatology. 2018 Jun; 18(5):S1424-3903(18)30605-7.

58 Fjeld K, Weiss FU, Lasher D, Rosendahl J, Chen JM, Johansson BB, et al. A recombined allele of the lipase gene CEL and its pseudogene CELP confers susceptibility to chronic pancreatitis. Nat Genet. 2015 May;47(5):51822.

59 Molven A, Fjeld K, Lowe ME. Lipase Genetic Variants in Chronic Pancreatitis: When the End Is Wrong, All's Not Well. Gastroenterology. 2016 Jun;150(7):1515-8.
60 Kukor Z, Mayerle J, Krüger B, Tóth M, Steed PM, Halangk W, et al. Presence of cathepsin $B$ in the human pancreatic secretory pathway and its role in trypsinogen activation during hereditary pancreatitis. J Biol Chem. 2002 Jun;277(24):21389-96.

61 Nijmeijer RM, van Santvoort HC, Zhernakova A, Teller S, Scheiber JA, de Kovel CG, et al.; Dutch Pancreatitis Study Group. Association analysis of genetic variants in the myosin IXB gene in acute pancreatitis. PLoS One. 2013 Dec;8(12):e85870.

62 Zenker M, Mayerle J, Lerch MM, Tagariello A, Zerres K, Durie PR, et al. Deficiency of UBR1, a ubiquitin ligase of the $\mathrm{N}$-end rule pathway, causes pancreatic dysfunction, malformations and mental retardation (Johanson-Blizzard syndrome). Nat Genet. 2005 Dec;37(12):1345-50.

63 Weiss FU, Schurmann C, Teumer A, Mayerle J, Simon P, Völzke H, et al. ABO blood type B and fucosyltransferase 2 non-secretor status as genetic risk factors for chronic pancreatitis. Gut. 2016 Feb;65(2):353-4.

64 Uc A, Perito ER, Pohl JF, Shah U, Abu-ElHaija M, Barth B, et al.; Consortium for the Study of Chronic Pancreatitis, Diabetes, and Pancreatic Cancer (CPDPC). INternational Study Group of Pediatric Pancreatitis: In Search for a CuRE Cohort Study: Design and Rationale for INSPPIRE 2 From the Consortium for the Study of Chronic Pancreatitis, Diabetes, and Pancreatic Cancer. Pancreas. 2018 Nov/Dec;47(10):1222-8.

65 Rosendahl J, Kirsten H, Hegyi E, et al. Genome-wide association study identifies inversion in the CTRB1-CTRB2 locus to modify risk for alcoholic and non-alcoholic chronic pancreatitis. Gut. 2018 Oct;67(10):1855-63.

66 Weiss FU. Pancreatic cancer risk in hereditary pancreatitis. Front Physiol. 2014 Feb;5: 70
67 Löhr JM, Faissner R, Koczan D, Bewerunge P, Bassi C, Brors B, et al. Autoantibodies against the exocrine pancreas in autoimmune pancreatitis: gene and protein expression profiling and immunoassays identify pancreatic enzymes as a major target of the inflammatory process. Am J Gastroenterol. 2010 Sep; 105(9):2060-71.

68 Pickartz T, Mayerle J, Lerch MM. Autoimmune pancreatitis. Nat Clin Pract Gastroenterol Hepatol. 2007 Jun;4(6):314-23.

69 Kamisawa T, Chari ST, Lerch MM, Kim MH, Gress TM, Shimosegawa T. Recent advances in autoimmune pancreatitis: type 1 and type 2. Gut. 2013 Sep;62(9):1373-80.

70 Beyer G, Menzel J, Krüger PC, Ribback S, Lerch MM, Mayerle J. [Autoimmune pancreatitis]. Dtsch Med Wochenschr. 2013 Nov; 138(46):2359-70. German.

71 Ferlay J, Shin HR, Bray F, Forman D, Mathers C, Parkin DM. Estimates of worldwide burden of cancer in 2008: GLOBOCAN 2008. Int J Cancer. 2010 Dec;127(12):2893-917.

72 Seufferlein T, Porzner M, Becker T, Budach V, Ceyhan G, Esposito I, et al.; Guidelines Programme Oncology AWMF; German Cancer Society eV; German Cancer Aid. [S3guideline exocrine pancreatic cancer]. Z Gastroenterol. 2013 Dec;51(12):1395-440. German.

73 Hruban RH, Takaori K, Klimstra DS, Adsay NV, Albores-Saavedra J, Biankin AV, et al. An illustrated consensus on the classification of pancreatic intraepithelial neoplasia and intraductal papillary mucinous neoplasms. Am J Surg Pathol. 2004 Aug;28(8):977-87.

74 Korc M. Driver mutations: a roadmap for getting close and personal in pancreatic cancer. Cancer Biol Ther. 2010 Sep;10(6):588-91.

75 Ellis I, Lerch MM, Whitcomb DC; Consensus Committees of the European Registry of Hereditary Pancreatic Diseases, Midwest MultiCenter Pancreatic Study Group, International Association of Pancreatology. Genetic testing for hereditary pancreatitis: guidelines for indications, counselling, consent and privacy issues. Pancreatology. 2001;1(5):405-15. 